\title{
Review
}

\section{I-wave Transcutaneous Thermotherapy for the Treatment of Benign Prostate Hypertrophy and Pelvic Dysfunction in Men}

\author{
Alain Bitton*, Sandrine E. A Fiol, Gérard Aknine \\ Urological Surgeon, Andrology and Men's Health Clinic, Switzerland
}

^Correspondence to: Alain Bitton; Men’s Health and Andrology Clinic - 95 Route de Florissant - 1206 Genève Switzerland; E-mail: drbitton@andrologue.com

Received: Dec 22 $2^{\text {nd }}, 2020$; Accepted: Dec 30 ${ }^{\text {th }}, 2020$; Published: Jan $7^{\text {th }}, 2021$

Citation: Bitton A*, Fiol S E. A, Aknine G. I-wave transcutaneous thermotherapy for the treatment of benign prostate hypertrophy and pelvic dysfunction in men. Urology Open A Open J. 2020; 2(1): 46-49. doi: 10.33169/uro.UOAOJ-2-112

\section{ABSTRACT}

I-wave heat therapy produces microwave-like energy using a high-frequency electromagnetic field to achieve an optimal hyperthermia effect on the targeted organs. One of the advantages of this non-invasive and effective method is that it uses the body's natural response to the application of heat to produce a desired therapeutic effect. This innovative, non-invasive and very promising method, which also requires no anesthesia, is performed in the practitioner's office on an outpatient basis. Regarding urological indications, it is Benign Prostate Hypertrophy and inflammatory prostatitis including pelvic dysfunction that will be mainly treated. However, many other indications are applicable and some are under evaluation. In this paper, we present a review of one of these techniques concerning the principle of i-wave external thermotherapy. In a separate article we will present our patient group and the first clinical results in Switzerland.
\end{abstract}

Keywords: Non-invasive external thermotherapy; Benign prostate; Hypertrophy; Safe and efficient treatment; Inflammatory prostatitis; Pelvic dysfunction.

\section{INTRODUCTION}

For many years, transurethral thermotherapy has been recognized as a safe and alternative method in the treatment of benign prostatic hyperplasia in men. ${ }^{1,2}$ Despite the minimally invasive aspect of the application of microwaves, some surgeons even used this technique for external application in obstructive patients with bladder catheters in whom a transurethral procedure could not be performed. ${ }^{3,4}$ The authors therefore report excellent and very promising results, which according to the studies would allow $65 \%$ of patients a significant improvement during 8 years after the application of external thermotherapy. ${ }^{4,5}$ Based on its results, many engineers and physicists have worked to develop a technique that can be safely used routinely applicable on an outpatient basis and that could provide quality functional results. Principles of i-wave Thermotherapy (Eugeniux)

I-wave heat therapy produces microwave-like energy using a high-frequency electromagnetic field to achieve an optimal hyperther- mia effect on the targeted organs. The radiofrequency type of energy generated by the applicator enables deep tissue treatment up to 15 $\mathrm{cm}$. The application of this energy allows the practitioner to maintain throughout the duration of the treatment a temperature which can oscillate between 39 and 44 degrees Celsius. The optimal and therapeutic temperature is obtained very quickly (from 8 to 10 minutes from the beginning of treatment) and is maintained constantly during the entire application of the radiofrequency. One of the advantages of this non-invasive and effective method is that it uses the body's natural response to the application of heat to produce a desired therapeutic effect. Throughout the treatment, the power generator is used to control and regulate the applied temperature in order to maintain a surface temperature 3.9 degrees Celsius lower (and therefore allowing safety) than the internal temperature.

\section{Therapeutic Indications}

I-wave transcutaneous thermotherapy conceptualized by its engineer 
Dr. G. Aknine from the company Eugeniux, makes it possible to apply efficiently and sequentially during a period of one hour a temperature of 44 degrees Celsius coupled with neuromodulation at the level of strategic and targeted organs in order to obtain a therapeutic response. This innovative, non-invasive and very promising method, which also requires no anesthesia, is performed in the practitioner's office on an outpatient basis. Regarding urological indications, we were able to consider so far Benign Prostate Hypertrophy (BPH) and inflammatory prostatitis including pelvic dysfunction. In the latter indications the results were good and the response of the patients in term of tolerance and satisfaction was very high. Thus, many other indications are applicable and some are under evaluation, allowing and opening a wide and interesting window of opportunity. (Table 1\&2)

Table 1. Below shows the many indications, non-exausthive, related to the application of i-wave transcutaneous thermotherapy

\begin{tabular}{|l|l|}
\hline Indications & Advantages \\
\hline BPH & Improvement of irritative symptoms \\
\hline Bladder retention & Removal of the catheter and / or reduction of the post-void residual \\
\hline Erectile dysfunction & $\begin{array}{l}\text { Improvement of erectile function by regulating blood flow to the perineum and the cavernous bodies. Reduction in the intake and dosage } \\
\text { of oral treatments (PDE5-inhibitors) }\end{array}$ \\
\hline Cystitis and prostatitis & Decreased prevalence of infections and improvement of inflammatory symptoms \\
\hline Libido & Regulation of androgen levels circulating by acting on the relaxation of prostate muscles \\
\hline Pelvic dysfunctions & Reduction of pain and tension by cellular regulation of muscles and fibroblasts \\
\hline $\begin{array}{l}\text { Testicular pain and tension Inflam- } \\
\text { matory epididymitis }\end{array}$ & Decreased congestion of the ducts and glands of the vas deferens and epididymis by regulation of the blood flow \\
\hline $\begin{array}{l}\text { Postoperative dysuria and / or ir- } \\
\text { ritative symptoms }\end{array}$ & Alleviation of symptoms and improvement of urine flow by regulating blood flow and reducing inflammation \\
\hline
\end{tabular}

Table 2. IPSS or Symptomatic Score of the Prostate to assess the degree of severity of symptoms related to benign prostatic hyperplasia

Q 1. In the past month, how often have you had the feeling that your bladder was not completely emptied after urinating?

0 points $=$ never

1 point = approximately 1 in 5

2 points = approximately 1 in 3

3 points = approximately 1 in 2

4 points = approximately 2 times out of 3

5 pts = almost always

Q 2. In the past month, how often did you need to urinate within 2 hours after having finished urinating?

0 points $=$ never

1 point = approximately 1 in 5

2 points = approximately 1 in 3

3 points $=$ approximately 1 in 2

4 points $=$ approximately 2 times out of 3

5 pts = almost always

Q 3. During the past month, how often have you had an interruption in the flow of urine, ie starting to urinate then stopping then restarting?

0 points = never

1 point = approximately 1 in 5

2 points = approximately 1 in 3

3 points = approximately 1 in 2

4 points = approximately 2 times out of 3

5 pts = almost always

Q 4. In the past month, after you felt the need to urinate, how often have you had difficulty holding on to urinate?

0 points $=$ never

1 point = approximately 1 in 5

2 points = approximately 1 in 3

3 points = approximately 1 in 2

4 points = approximately 2 times out of 3

5 pts = almost always

Q 5. During the past month, how often have you had a decrease in the size or strength of the urine stream?

0 points $=$ never

1 point = approximately 1 in 5

2 points = approximately 1 in 3

3 points $=$ approximately 1 in 2 


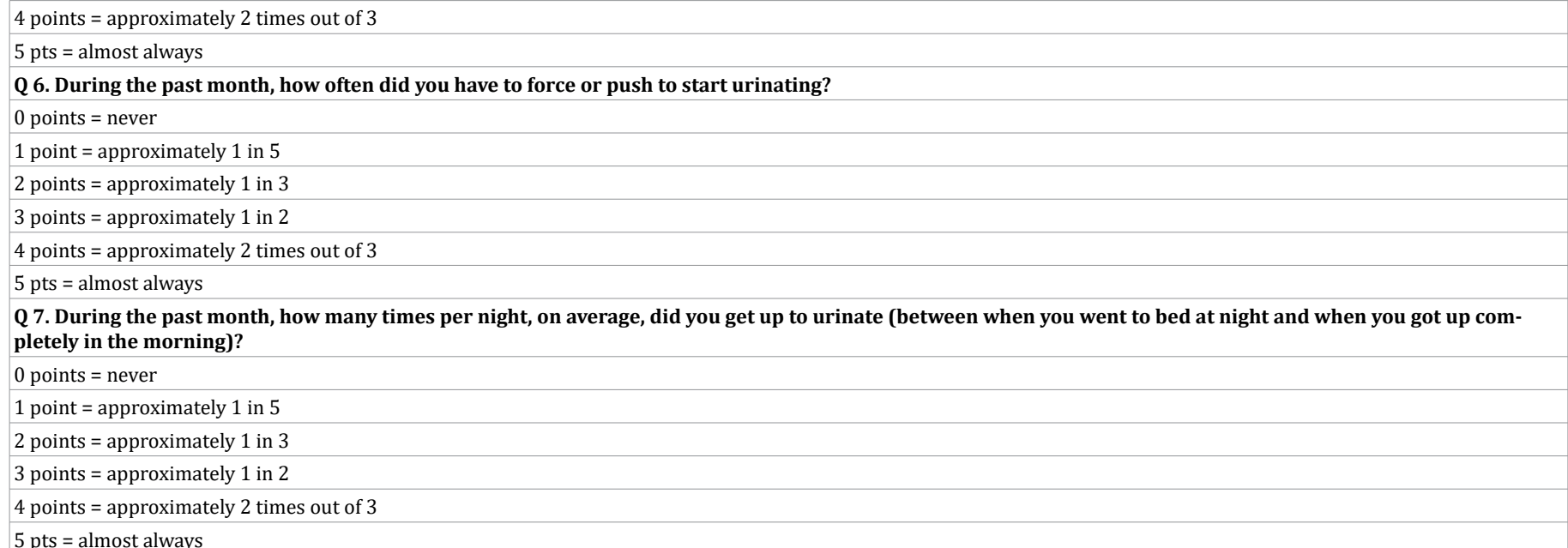

\section{Recruitment of Patients for Therapy}

After a careful history and prostate examination, patients are selected for therapy based on clinical symptoms on one hand and the I-PSS score (prostatic symptom score) on the other hand. Additional examinations such as free flowmetry, urethrocysoscopy or urodynamic evaluation are only performed in doubtful cases or in order to clarify the diagnosis. To date, we can list the indications of benign non-obstructive prostatic hyperplasia in patients presenting mainly irritative symptoms as well as inflammatory prostatitis and pelvic dysfunction as the major indications for this type of treatment. Indeed, these pathologies respond best to the application of $\mathrm{i}$-wave heat therapy. In addition, some patients who have been operated on for transurethral resection of the prostate (by ND- Yag Laser, Holmium or Plasma Edge) for obstructive BPH and presenting post-operative irritative and inflammatory symptoms, also respond very well to a few treatment sessions. allowing to spare anti-inflammatory treatments or corticosteroids.

Depending on the intensity of the IPSS score, patients are scheduled for 6 to 8 treatment sessions, or even more, if necessary. Depending on the progress, some patients may repeat the sessions after a few months of break.

The IPSS Score is calculated by adding the scores obtained by answering the 7 questions: Depending on the score, a severity scale can be established:

\section{0 - 7 Little symptomatic \\ 8 - 19 Moderately symptomatic \\ 20 - 35 Severe symptoms}

This score, although very simplistic, but very useful in current urology practice, makes it possible to identify patients according to their symptoms and especially to follow the progress of therapy.

On the day of treatment, the patient completes an IPPS score to monitor the progression of symptoms and the progression of treatment effects. The patient also performs a flow measurement and a measurement of the post-voiding residual before the start of the session.

For the follow-up, apart from the clinical and ultrasound evaluation of the prostate, we suggest performing a control flow measurement as well as blood PSA assay. These tests are carried out at 3 and 6 months allowing progress to be monitored.

\section{Course of Therapy}

During therapy, which lasts in principle one hour of time, the patient is comfortably installed in a chair (Figures $1 \mathrm{a}-1 \mathrm{~b} \& 2$ ) allowing the pads to be put in place including the external electrodes (digital multipoint temperature sensors) connected to the treatment console. These applicators are ultra-flexible and comfortable and allow heat distribution to a depth of $15 \mathrm{~cm}$ at tissue level. During the session, the specialist can at any time activate neuromodulation to reduce the sensation of pain due to the rise in temperature. This non-invasive, real-time temperature control with autofocus system is a real gain in energy application and therapeutic safety control.

Figure 1a\&1b. Console and monitor with electrodes connected externally to the pelvicprostatic area and the bladder area
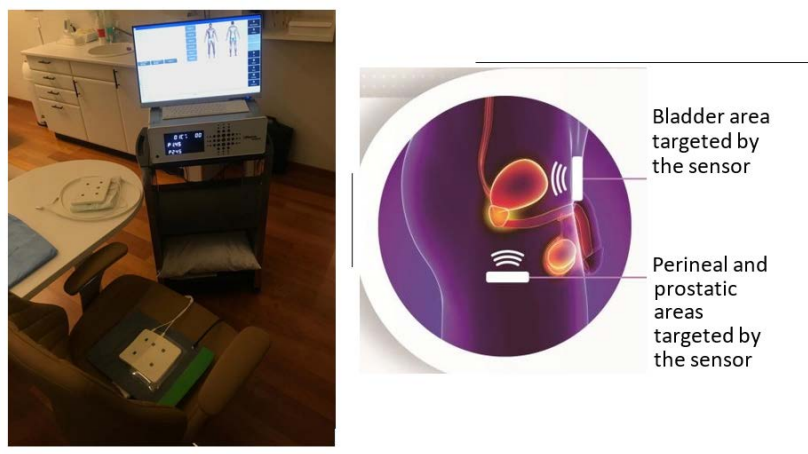

Figure 2. Patient positioning showing the course of the session with an automatic biological tissue adaptation system and maintaining a low skin temperature

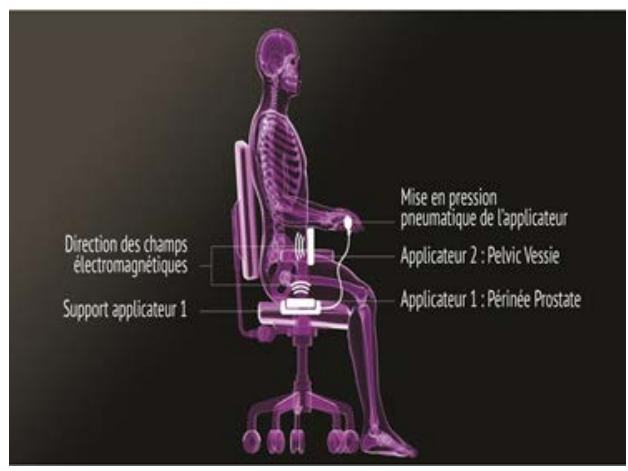




\section{Promising Preliminary Results}

As we have already specified, the purpose of this article is above all to present this innovative and non-invasive method that we have been using in our clinic since March 2019. To date, several hundred patients have been treated with excellent functional and very promising results.

In a future article we will present our first clinical results in Switzerland. Over time, we were able to refine our indications and the choice of patients eligible for therapy as well as the optimal number of sessions for each indication.

\section{CONCLUSION}

I-wave heat therapy is an innovative, non-invasive and very promising method of treatment for benign prostatic patholgies using molecular thermal wave therapy and providing excellent functional results. Indeed, this molecular therapy system coupled with ultra-high radiofrequencies allowing optimal penetration into biological tissues helps to improve tissue trophicity and to regulate blood circulation in order to obtain an optimal therapeutic effect. In addition, a low frequency modulation close to the heart rate of the main wave beam allows hyper-vascularization. This gives the therapy the ability to improve blood flow optimally when reaching the therapeutic 44 degrees Celsius. Thus, even if transurethral thermotherapy remains an excellent alternative in non-invasive surgery for $\mathrm{BPH}[5,6]$ and other benign prostate pathologies, external transcutaneous application thanks to the i-wave form gives a new and very promising dimension. This new application of the thermotherapy in the treatment of benign prostatic conditions can be safely done in a one day clinic without any kind of anesthesia and completely painless and convenient for the patient.

\section{CONFLICTS OF INTEREST}

None.

\section{REFERENCES}

1. Devonec M. Prog Urol. 2002; 12: 1330-1340

2. Bitton A, Thalmann GN, Burkhard F, Graber SF, Grünig O, Studer UE. Progrès en Urologie. Vol. 6, 1999.

3. Yerushalmi A, Shani A, Fishelovitz Y, Arielly J, Singer D, Levy ZE.et al. Local Microwave Hyperthermia in the Treatment of Carcinoma of the Prostate. Oncology. 1986; 43: 299-305.

4. Yerushalmi A, Singer D, Katsnelson R, Levy E, Shani A. Localised Deep Microwave Hyperthermia in the Treatment of Benign Prostatic Hyperplasia: Long-term Assessment. British Journal of Urology. 1992; 70: $178-182$

5. Servadio C, Lindner A, Lev A, Leib Z, Siegel Y, Braf S. Further observations on the effect of local hyperthermia on benign enlargement of the prostate. World J. Urol. 1989; 6: 204.

6. Steg A, Zerbib M, Conquy S, Maugenest JP. Fournier's gangrene developing secondary to infected hydrocele: A unique clinical scenario. Ann Urol. 1990; 24(2): 131-134. 